Check for updates

Cite this: Nanoscale Adv., 2019, 1, 969

\title{
Controlled disassembly of a DNA tetrahedron using strand displacement $\uparrow$
}

\author{
Arun Richard Chandrasekaran (D)* and Ken Halvorsen*
}

Received 11th November 2018

Accepted 21st December 2018

DOI: $10.1039 / \mathrm{c} 8 \mathrm{na00340h}$

rsc.li/nanoscale-advances

In this study, we assembled a DNA tetrahedron containing single stranded extensions in the middle of the struts. Using these extensions as toeholds, the tetrahedron can be disassembled by nucleic acid triggers via strand displacement. The release mechanism is sequence specific, is functional in biological fluids such as serum and urine, and the kinetics of the disassembly process can be controlled by different molar ratios of the release strand. Such DNA nanostructures that respond to external stimuli have potential use in biosensing and drug delivery, and we demonstrate a proof-of-concept of this approach for microRNA detection.

In a 1999 episode of X-Files, Fox Mulder races to find a cure for his boss' condition, and is told that it is "technology that the world believes is purely theoretical", to which he exclaims "nanotechnology". Two decades later, the application of nanotechnology to health and medicine has moved beyond the theoretical. While we are still a far cry from the complex, selfreplicating, and autonomous machines presented in science fiction, scientists have created simple nanomachines in the lab. Various versions have been based on metal nanowires, carbon nanotubes, artificial biological assemblies, small molecule motifs and mechanically interlocked molecules. ${ }^{\mathbf{1 , 2}}$ DNA is one such material that is increasingly used as a building block for the bottom-up construction of a wide variety of nanostructures. ${ }^{3,4}$ The programmability of these structures allows greater control over defined assembly and response to chemical and molecular cues. ${ }^{5-7}$ Specifically, hollow or wireframe DNA nanostructures $^{8}$ can encapsulate guest objects such as proteins, ${ }^{9}$ fluorescent biopolymers, ${ }^{\mathbf{1 0}}$ quantum $\operatorname{dots}^{\mathbf{1 1}}$ and nanoparticles, ${ }^{12}$ with their target application being on-demand cargo delivery. ${ }^{13}$ To achieve this, DNA nanostructures are designed to respond to chemical or environmental cues such as other biomolecules, ${ }^{14}$ light, ${ }^{15} \mathrm{pH}^{16}$ or enzymes. ${ }^{17}$ These cues

The RNA Institute, University at Albany, State University of New York, Albany, NY, USA.E-mail: arun@albany.edu; khalvorsen@albany.edu

$\dagger$ Electronic supplementary information (ESI) available. See DOI: 10.1039/c8na00340h affect the self-assembled DNA nanostructures leading to conformational changes such as pore size widening, ${ }^{14}$ unlinking of cargo, ${ }^{15}$ or complete dissociation,,${ }^{16,17}$ all of which result in cargo release. Here we present a DNA tetrahedron that can be controllably disassembled via toehold-based strand displacement process ${ }^{18}$ that is activated upon binding to a specific nucleic acid trigger.

The DNA tetrahedron used in this study is assembled from three-point-star motifs that connect to each other via programmed sticky ends (Fig. 1a). ${ }^{19}$ The motif contains one long, threefold-repeating strand (L, colored orange in Fig. 1a), three copies of medium strands (M, cyan), and three copies of short, peripheral strands ( $\mathrm{S}$, red-and-dark blue). The center portion of the motif contains $5 \mathrm{~T}$ loops (on strand L) that provide flexibility for the arms to associate into higher order structures (Fig. S1 $\dagger$ ). Each arm of the motif contains two helices connected by a single crossover. Both helices of the motif are tailed with 7-nt sticky ends (one extending from $\mathrm{S}$ and one extending from $\mathrm{M}$ ) so that motifs can join through sticky end connections to further assemble into the DNA tetrahedron (a tetrahedron contains four units of the motif). The edges of the assembled tetrahedron are four turns of DNA $(\sim 14 \mathrm{~nm})$. The resulting tetrahedron is robust and remains stable at room temperature for a prolonged time (Fig. S2†).

To enable controlled disassembly of our tetrahedron, we modified the design by extending the single-stranded overhang on strand $\mathrm{S}$ by 8 nucleotides beyond the sticky end region (dark blue region of strand $S$ in Fig. 1a). Upon assembly, the DNA tetrahedron contains single-stranded tails protruding from the middle of the struts that act as toeholds to recognize external nucleic acid triggers. The release strand ( $\mathrm{R}$, dark blue-red) is designed to be complementary to the toehold region and to the remainder of strand S. Addition of the release strand results in strand displacement and elimination of the sticky end interactions that hold the tetrahedron together, leading to disassembly of the DNA tetrahedron into its component motifs (Fig. 1b). 

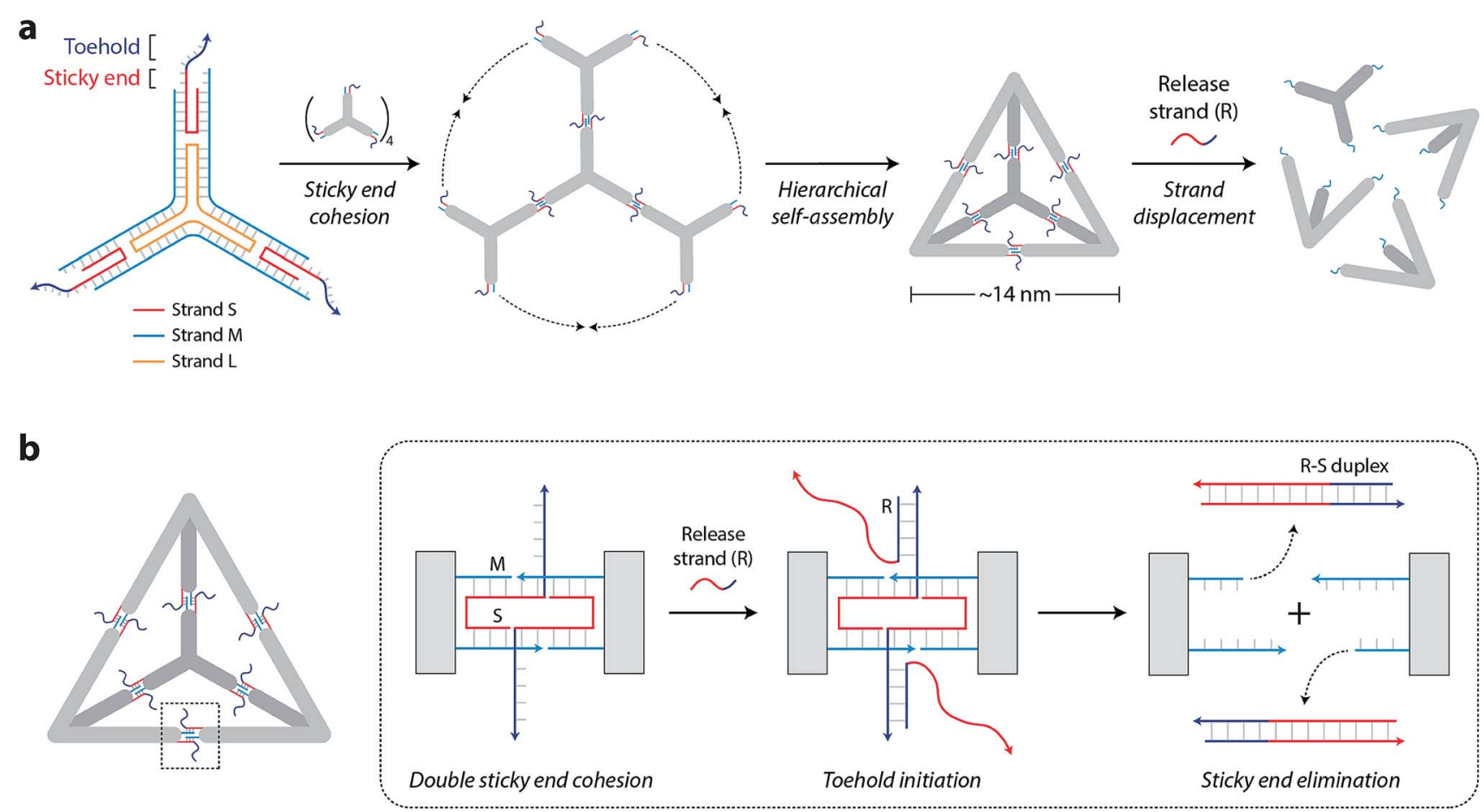

Fig. 1 Design of assembly and toehold-activated release. (a) Scheme showing the assembly and disassembly of a DNA tetrahedron from threepoint-star motifs. The motif is tailed with single-stranded extensions that act as toeholds to initiate disassembly on the addition of a release strand. (b) The release strand $\mathrm{R}$ is complementary to strand $\mathrm{S}$, and the toehold region (dark blue region on strand S) initiates the strand displacement process. Due to the inherent symmetry of the motif, strand displacement occurs at sticky regions on both helices in each arm of the motif. This process leads to removal of the short strand from the tile, resulting in elimination of sticky ends and thus disassembly of the tetrahedron into its component motifs.

We assembled the DNA tetrahedron by mixing strands L, M, and $\mathrm{S}$ in a ratio of $1: 3: 3$ in $\mathrm{Mg}^{2+}$-containing tris-acetate-EDTA (TAE) buffer and slowly annealing the solution from $90{ }^{\circ} \mathrm{C}$ to $4{ }^{\circ} \mathrm{C}$. This one-step annealing forms the individual three-pointstar motifs which further self-assemble to form the DNA tetrahedron. We validated the formation of our toehold modified DNA tetrahedron (TET) using polyacrylamide gel electrophoresis (Fig. 2a, lane 4), a technique well-established to analyze such DNA nanostructures. ${ }^{19}$ The introduction of singlestranded toeholds does not seem to affect the self-assembly of the tetrahedron from three-point-star motifs (Fig. S3†). By adding the release strand, we confirmed that the tetrahedron is disassembled into its component motifs (Fig. 2a, lane 5). Addition of the release strand did not have any effect on a tetrahedron without a toehold, confirming that disassembly proceeds by toehold-mediated strand displacement (Fig. S4 $\dagger$ ).

To further investigate the disassembly process, we next measured the progression of disassembly at different time points for different concentrations of the release strand $(1: 2$, $1: 5$ or $1: 10$ molar ratios of tetrahedron : release strand) (Fig. 2b, full gels in Fig. S5 $\dagger$ ). To better visualize the kinetics of disassembly, we calculated the conversion by quantifying the

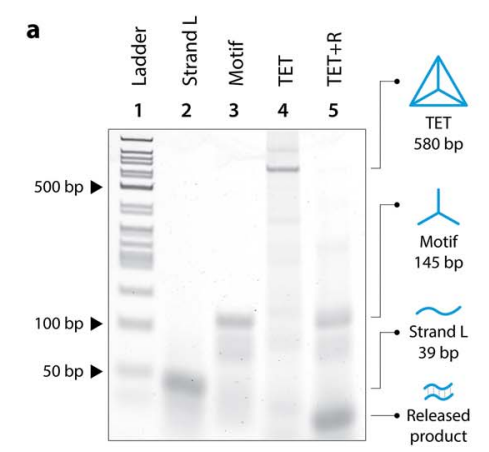

b

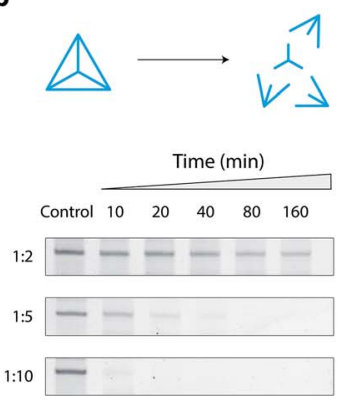

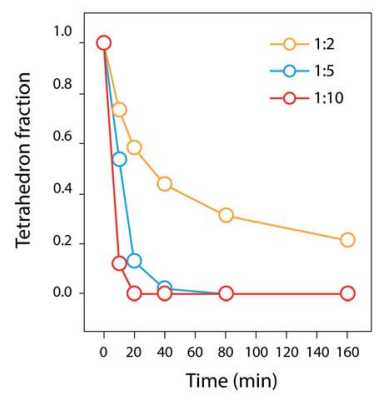

Fig. 2 Assembly of tetrahedron and disassembly kinetics. (a) Polyacrylamide non-denaturing gel showing the formation of the tetrahedron (lane 4) and its disassembly on the addition of the release strand (lane 5). (b) Gels showing disassembly of the tetrahedron at various molar ratios of the release strand and at different time points. (c) Quantitative analysis of tetrahedron disassembly. 


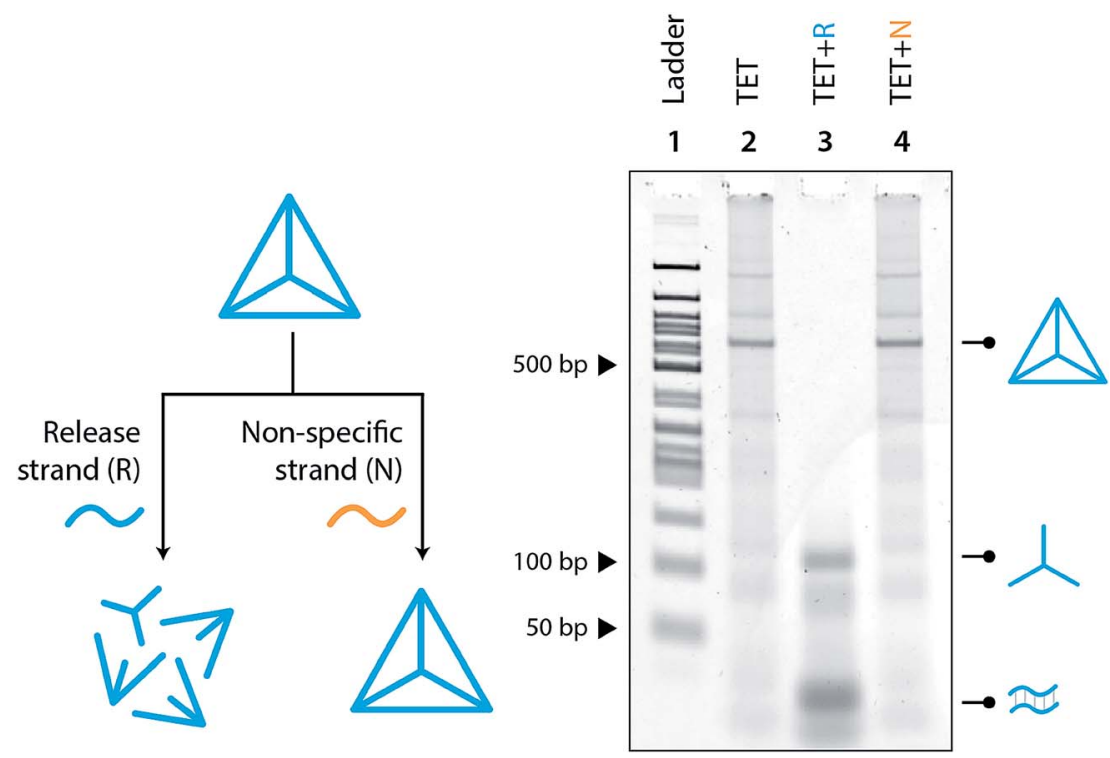

Fig. 3 Specificity of release mechanism. Polyacrylamide non-denaturing gel showing the disassembly of the tetrahedron only on the addition of the correct release strand (lane 3) and an incorrect release strand has no effect (lane 4).

band intensity of the tetrahedron at different time points (Fig. 2c). Results show that the release mechanism can be effective in as little as 10 minutes for $1: 10$ molar ratio. The kinetics of the release is also affected by the toehold length, with 8 nucleotides providing the fastest disassembly compared to shorter toeholds (Fig. S6†).

Moving toward biological applications, we investigated the specificity of the release mechanism and compatibility with biological fluids. To test the specificity, we used a non-specific release strand at a $1: 10$ ratio and showed that the tetrahedron is not disassembled under those conditions (Fig. 3). We further tested the specificity by using a release strand that contains a single nucleotide mismatch in the toehold region (Fig. S7 $\dagger$ ). Results showed that the tetrahedron was not disassembled by a release strand with even a single nucleotide mismatch. Extending this concept beyond a single off-target release strand, we challenged the tetrahedron disassembly process by testing in Fetal Bovine Serum (FBS) and in synthetic urine. In both cases, the tetrahedra maintained stability in these fluids and only disassembled in the presence of the release strand (Fig. 4).

The features of our tetrahedron disassembly approach could be useful for various nucleic acid sensing applications. One such area of interest is detection of microRNAs, small RNA fragments (sometimes similar in sequence) that are increasingly recognized for their roles in gene regulation and as disease biomarkers. ${ }^{20,21}$ To demonstrate this as a potential biological application of our approach, we redesigned the tetrahedron to be disassembled upon recognition of a specific microRNA. The resultant tetrahedron had a lower yield (Fig. S8 $\dagger$ ) than our native tetrahedron, likely due to sensitivity to component sequences that were optimized for self-assembly in the native structure. Even with this lower yield, however, we showed that the tetrahedron was successfully disassembled in the presence of the correct microRNA. Disassembly was progressively reduced but not completely eliminated when presented with microRNA variants that differ by $1 \mathrm{nt}$ and $2 \mathrm{nt}$.

Stimuli-responsive DNA nanostructures are increasingly shown to be useful in biosensing and drug delivery applications. The inclusion of a toe-hold is a programmable feature, and via strand displacement, allows reconfiguration of small DNA objects, ${ }^{22}$ nanodevices ${ }^{23}$ or larger DNA origami structures. ${ }^{24}$ In this work, we show that designed single-stranded

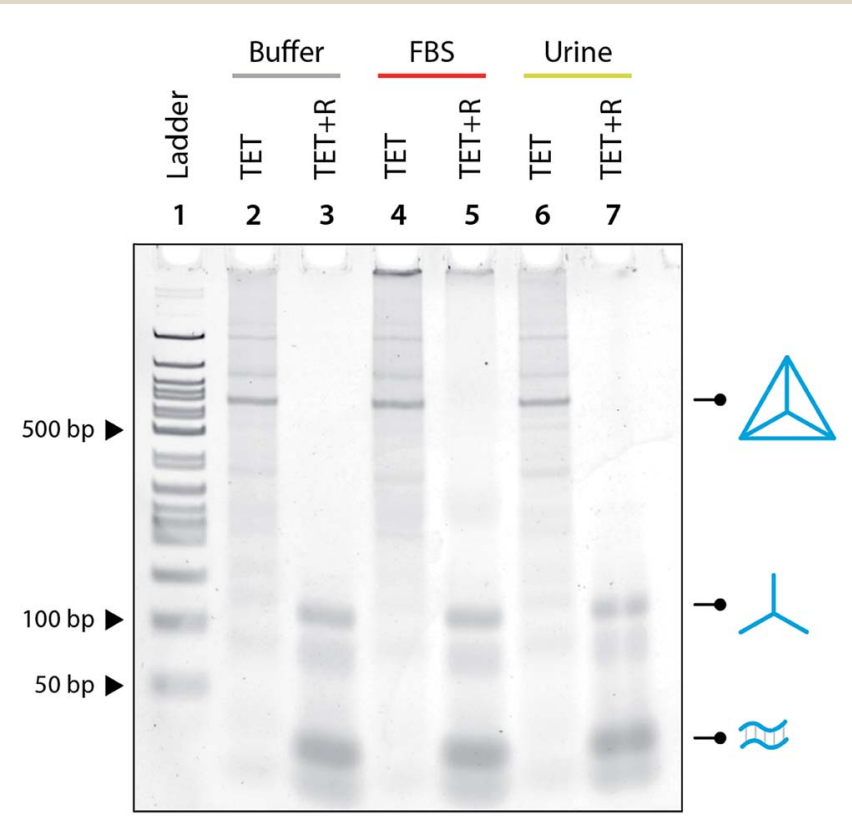

Fig. 4 Biostability of construction and release. The DNA tetrahedron is stable in $10 \%$ FBS and in synthetic urine and the release mechanism is fully functional in both biofluids on addition of release strand and incubation for 1 hour. 
extensions in DNA tetrahedra can be used as toeholds to controllably disassemble the nanostructure into its component units. This concept could be extended to other DNA polyhedra ${ }^{19}$ and possibly combined with macromolecular sensing strategies. Our preliminary data shows that tetrahedra can be designed to recognize microRNAs, adding to the existing suite of DNA nanotechnology biosensors. ${ }^{25-28}$ Since the tetrahedron and other polyhedra contain internal cavities that could be loaded with cargo, our strategy also has the potential to be used for drug delivery platforms. Considering the demonstrated stability in biological fluids and the ability to be triggered open on recognizing biologically relevant nucleic acids, these structures show promise in the creation of "sense-and-act" nanomachines.

\section{Conflicts of interest}

The authors have no conflicts to declare.

\section{Acknowledgements}

Research reported in this publication was supported by the NIH through NIGMS under award R35GM124720 to K. H.

\section{References}

1 G. A. Ozin, I. Manners, S. Fournier-Bidoz and A. Arsenault, Adv. Mater., 2005, 17, 3011-3018.

2 S. Erbas-Cakmak, D. A. Leigh, C. T. McTernan and A. L. Nussbaumer, Chem. Rev., 2015, 115, 10081-10206.

3 P. L. Xavier and A. R. Chandrasekaran, Nanotechnology, 2018, 29, 062001.

4 A. R. Chandrasekaran, N. Anderson, M. Kizer, K. Halvorsen and X. Wang, ChemBioChem, 2016, 17, 1081-1089.

5 X. Qu, D. Zhu, G. Yao, S. Su, J. Chao, H. Liu, X. Zuo, L. Wang, J. Shi, L. Wang, W. Huang, H. Pei and C. Fan, Angew. Chem., Int. Ed., 2017, 56, 1855-1858.

6 X. Qu, S. Wang, Z. Ge, J. Wang, G. Yao, J. Li, X. Zuo, J. Shi, S. Song, L. Wang, L. Li, H. Pei and C. Fan, J. Am. Chem. Soc., 2017, 139, 10176-10179.

7 W. Lai, L. Ren, Q. Tang, X. Qu, J. Li, L. Wang, L. Li, C. Fan and H. Pei, ACS Nano, 2018, 12, 7093-7099.

8 A. R. Chandrasekaran and O. Levchenko, Chem. Mater., 2016, 28, 5569-5581.

9 C. Zhang, C. Tian, F. Guo, Z. Liu, W. Jiang and C. Mao, Angew. Chem., Int. Ed., 2012, 51, 3382-3385.

10 D. Bhatia, S. Surana, S. Chakraborty, S. P. Koushika and Y. Krishnan, Nat. Commun., 2011, 2, 339.
11 D. Bhatia, S. Arumugam, M. Nasilowski, H. Joshi, C. Wunder, V. Chambon, V. Prakash, C. Grazon, B. Nadal, P. K. Maiti, L. Johannes, B. Dubertret and Y. Krishnan, Nat. Nanotechnol., 2016, 11, 1112-1119.

12 Y. Li, Z. Liu, G. Yu, W. Jiang and C. Mao, J. Am. Chem. Soc., 2015, 137, 4320-4323.

13 B. R. Madhanagopal, S. Zhang, E. Demirel, H. Wady and A. R. Chandrasekaran, Trends Biochem. Sci., 2018, 43, 9971013.

14 C. Zhang, C. Tian, X. Li, H. Qian, C. Hao, W. Jiang and C. Mao, J. Am. Chem. Soc., 2012, 134, 11998-12001.

15 R. E. Kohman, S. S. Cha, H. Y. Man and X. Han, Nano Lett., 2016, 16, 2781-2785.

16 Z. Liu, Y. Li, C. Tian and C. Mao, Biomacromolecules, 2013, 14, 1711-1714.

17 Y. Ma, Z. Wang, Y. Ma, Z. Han, M. Zhang, H. Chen and Y. Gu, Angew. Chem., Int. Ed., 2018, 57, 5389-5393.

18 B. Yurke, A. J. Turberfield, A. P. Mills Jr, F. C. Simmel and J. L. Neumann, Nature, 2000, 406, 605-608.

19 Y. He, T. Ye, M. Su, C. Zhang, A. E. Ribbe, W. Jiang and C. Mao, Nature, 2008, 452, 198-201.

20 H. Dong, J. Lei, L. Ding, Y. Wen, H. Ju and X. Zhang, MicroRNA: function, detection, and bioanalysis, Chem. Rev., 2013, 113, 6207-6233.

21 D. P. Bartel, MicroRNAs: genomics, biogenesis, mechanism, and function, Cell, 2004, 116, 281-297.

22 R. P. Goodman, M. Heilemann, S. Doose, C. M. Erben, A. N. Kapanidis and A. J. Turberfield, Nat. Nanotechnol., 2008, 3, 93-96.

23 A. R. Chandrasekaran, O. Levchenko, D. S. Patel, M. MacIsaac and K. Halvorsen, Nucleic Acids Res., 2017, 45, 11459-11465.

24 E. S. Andersen, M. Dong, M. M. Nielsen, K. Jahn, R. Subramani, W. Mamdouh, M. M. Golas, B. Sander, H. Stark, C. L. Oliveira, J. S. Pedersen, V. Birkedal, F. Besenbacher, K. V. Gothelf and J. Kjems, Nature, 2009, 459, 73-76.

25 A. R. Chandrasekaran, M. MacIsaac, P. Dey, O. Levchenko, L. Zhou, M. Andres, B. K. Dey and K. Halvorsen, Sci. Adv., 2018, DOI: $10.1126 /$ sciadv.aau9443.

26 M. Xiao, A. R. Chandrasekaran, W. Ji, F. Li, T. Man, C. Zhu, X. Shen, H. Pei, Q. Li and L. Li, ACS Appl. Mater. Interfaces, 2018, 10, 35794-35800.

27 M. Rana, M. Balcioglu, M. Kovach, M. S. Hizir, N. M. Robertson, I. Khan and M. V. Yigit, Chem. Commun., 2016, 52, 3524-3527.

28 M. Lin, P. Song, G. Zhou, X. Zuo, A. Aldalbahi, X. Lou, J. Shi and C. Fan, Nat. Protoc., 2016, 11, 1244-1263. 